\title{
Multi-Robot Perimeter-Shaping through Mediator-Based Swarm Control
}

\author{
Shin-Young Jung and Michael A. Goodrich \\ Computer Science \\ Brigham Young University \\ Provo, Utah, USA \\ ldsrogan@byu.edu
}

\begin{abstract}
A swarm is a group of uninformed individuals that exhibit collective behaviors. The group without any information has limited ability to achieve complex goals. Human-swarm interaction methods often allow a human to influence these uninformed individuals through either leadership or predation as informed agents that directly interact with humans. These methods of influence have two main limitations: (1) although leaders sustain influence over nominal agents for a long period of time, they tend to cause all collective structures to turn in to flocks (negating the benefit of other swarm formations) and (2) predators tend to cause collective structures to fragment. In this paper, we present the use of mediators as a novel form for humanswarm influence and use mediators to shape the perimeter of a swarm. The mediator method uses special agents that operate from within the spatial center of a swarm. This approach allows a human operator to coordinate multiple mediators to modulate a rotating torus into various shapes while sustaining influence over the swarm, avoiding fragmentation, and maintaining the swarm's connectivity. The use of mediators allows a human to mold and adapt the torus' behavior and structure to a wide range of spatio-temporal tasks such as military protection and decontamination tasks. This paper also provides the results of the experiment concerned with decontamination task that compares previous informed agent methods to the mediator-based control with regard to manageability and performance.
\end{abstract}

\section{INTRODUCTION}

Performing tasks with multiple robots potentially increases the performance and flexibility of robots, but at a cost of increased difficulty for the human(s) responsible for managing the robots. To manage multiple robots efficiently, many researchers have tried to find an efficient control method by observing swarm behavior in nature. In this work, a swarm consists of a group of simple individuals who act without input from a centralized controller, responding spontaneously to signals from its environment and its neighbors.

In this paper, we utilize a swarm model in which each individual in the swarm follows three simple heuristic rules [1][3]: (1) each individual attempts to stay within a certain range of its neighbors, (2) each individual tries to avoid collisions by maintaining a minimum distance from its neighbors, and (3) each individual matches its velocity to its neighbors. Swarms that follow these rules can exhibit a range of mobile spatial structures including simple flocking and torus behaviors similar to what have been observed in nature for groups of birds or fish. Although there are a range of collective structures that can be produced by these simple rules, we focus on a torus structure, partly because of its usefulness and partly because it has received less attention in the literature than other swarm structures.

A swarm that is formed by only these simple rules has limited information and, consequently, it is hard to shape and guide the way the resulting swarm structures move. One way to allow a swarm to achieve complex goals and adapt to changing environments is to increase the complexity and sophistication of the individual agents. Another way, which minimizes the complexity of individual agents, is to enable a human to interact with the swarm. Both approaches have been used in the literature and have been applied to important problems such as military force protection, firefighting, search and rescue, etc. [4], [5].

In this paper, we adopt the latter approach and influence swarm behavior by placing informed agents among uninformed agents. The informed agents directly respond to an operator's input, and the uninformed agents respond to the informed agents. In this way, a human can use direct influence over a handful of robots to control an entire swarm.

We introduce a novel way to control a swarm by applying persistent influence with a new type of informed agents called mediators. The special types of mediators that we describe below repel uninformed agents similar to the way a predator does [6], [7] but use parameters that cause the uninformed agents to "stay close but not too close" to the mediators. This approach allows a human to alter the collective shape exhibited by agents as they encircle a group of mediators. In addition, the mediator-based control increases the manageability of a human's influence over swarms compared to leaders and predators. We investigate these claims about swarm manageability and performance using a study of a decontamination task with three different approaches to informed agent-based control.

\section{RELATED WORK}

Previous work on Human Swarm Interaction (HSI) has described several different methods for controlling the agents in a swarm. Kira and Potter used virtual leaders to influence a swarm [8]. Similar to their control method, Olfati-Saber also used a virtual leader to control the behavior of a flock [1]. 
$\mathrm{Su}$ et al. proposed a method for controlling a flock using multiple virtual leaders where agents have limited sensing capabilities [9]. Mabrouk et al. use a virtual leader to escape from a local minima in a reactive problem domain [10]. While these papers deal with enabling human interaction with swarms through virtual leaders, our approach adds human interaction to a swarm through one or more informed agents that simultaneously attract and repel uninformed agents to shape and steer a torus.

Elkaim and Kelbley showed formation shapes that are similar to those that we propose. They used virtual leaders that exerted attraction on other agents, and allowed obstacles to exert repulsion potential forces on those agents [11]. The basic concept of their approach is to maintain an equilibrium between inter-agent potential forces and the forces applied by a virtual leader positioned at the centroid of the agents. Our approach differs in that it does not account for obstacles and uses mediators that exert only a repulsion force on nominal agents.

Varghese and McKee manipulate agent position by calculating a geometric transformation that makes each agent move to a desired position while avoiding collisions with obstacles [12]. Kawashima et al. investigate the responsiveness of fixed-communication leader-follower networks for manipulating multi-agent formations [13]. Our model does not require calculating a geometric transformation or a fixed communication topology but, instead, agents are able to flexibly form various formations through the influence of mediators.

Kolling et al. present two different ways of enabling human operators to control robot swarms: selection control and beacon control [14]. Selection control allows the operator to select a subset of agents in the swarm and to control consistently. This is a form of direct and intermittent interaction that requires that the operator knows the entire environment. Beacon control is similar to a leader or a predator approach, but one in which the leader/predator doesn't move. This is a form of indirect interaction. In contrast to these approaches, control by mediator agents uses direct interaction and includes both parameter setting and persistent influence.

\section{THE MODEL}

The model we propose is biomimetic, meaning biologically inspired [2]. Fig. 1 shows how agents in nature simultaneously respond to both their neighbors and to predators, producing round empty spaces around the predators. Inspired by this natural behavior, we propose two different agent types, nominal agents and mediators, and a mechanism for combining these two agent types. Mediators are directly influenced by a human, and nominal agents are influenced directly by mediators. The term mediator indicates that the human does not directly influence the nominal agents, but rather indirectly influences nominal agents via the mediators. This means that the human and nominal agents share control over the specific structure of the swarm because the human can influence nominal agents only by appropriately managing mediators.

We adopt a switching-based control model in which the nominal agents either $(a)$ react to their neighbors or $(b)$ react to the mediators but $(c)$ not both. The human provides input to the

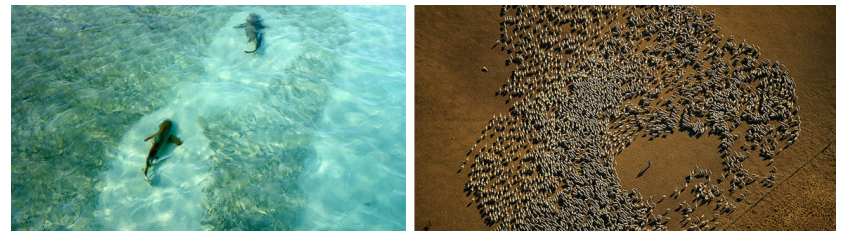

Fig. 1. Predators with agents in nature.

mediators, and the mediators influence nominal agents that are within range of the mediators. Since the nominal agents may move in and out of the sensing range of the mediator, agents can switch back and forth between inter-agent influence and mediator influence. Because avoiding collisions is critical, we also added a switch in which nominal agents ignore mediators if inter-agent distance drops below a threshold.

\section{A. Nominal Agent}

A nominal agent is a typical type of an uninformed agent that uses a two-dimensional implementation of Couzin's three dimensional model [2]. Since we are interested in ground robots, the two-dimensional model is sufficient. As mentioned previously, this model uses three basic rules and can produce two fundamentally different structures: a torus and a flock [2], [3]. The first rule is that each agent attempts to stay close to other agents. This is accomplished by the zone of attraction $\left(R^{a t t}\right)$. Agents are attracted to neighbors within the zone of attraction to maintain swarm connectivity. The second rule is that each agent tries to avoid collisions with other agents by maintaining a minimum inter-agent distance. This is accomplished by the zone of repulsion $\left(R^{r e p}\right)$. This rule has the highest priority [2], meaning an agent ignores attraction and orientation forces in order to avoid a neighbor within its zone of repulsion. The third rule is that each agent matches its velocity and direction with its neighbors. This is accomplished by the zone of orientation $\left(R^{\text {ori }}\right)$.

In addition to Couzin's rules, we add another rule that dictates how the nominal agents behave when near a mediator. In particular, we assume that when nominal agents are near a mediator they ignore all inter-agent influences except repulsion and respond only to the mediator. This means that nominal agents need two sets of parameters for determining their actions: a set of parameters for when they are in the presence of a mediator, and a set of parameters for when they are not in the presence of a mediator. It is useful to treat the former set as a property of the mediator rather than the nominal agent. This allows us to systematically explore how nominal agents respond to the mediator, which we now explain further.

\section{B. Mediator}

For the purposes of this paper, leadership means means that a mediator (called a leader in this case) exerts an attractive influence over uninformed agents, pulling uninformed agents toward them. Conversely, predation means the mediator (called a predator in this case) exerts a repelling influence over uninformed agents, pushing uninformed agents away. Thus, we have two specific types of mediators that we refer to as leaders and predators. We now introduce a third type of mediator, which we will call the mediator and distinguish it from leaders and predators. 
The mediator influences nominal agents using only predation, but uses what we can call a "weak" form of predation. Weak predation means the mediator repels nominal agents, but the zone of mediator predation is smaller than the zone of nominal attraction. Let the zone of predation be denoted by $R^{\text {pred }}$. Weak predation occurs when $2 \times R^{\text {pred }}<R^{\text {att }}$, or equivalently $R^{\text {pred }}<R^{\text {att }} / 2$, which means that the range of influence between nominal agents exceeds the maximum range of mediator influence on the

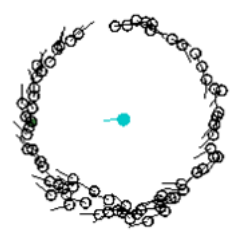

Fig. 2. Torus behavior around the mediator. nominal agents. This allows nominal agents to stay in a cohesive torus formation when a mediator is in the center of the group. Combining this constraint with parameters that Couzin used to produce a torus yields the following ordering of parameters:

$$
R^{\text {rep }} \leq R^{\text {ori }}<R^{\text {pred }}<R^{\text {att }} / 2 .
$$

This allows a mediator to be in the middle of a torus and "steer" the torus in various directions, as shown in Fig. 2.

Note that this means that the nominal agents use the attraction, orientation, and repulsion behaviors identified in the previous section when not in the presence of a mediator; when a mediator is nearby, the mediator repels the agents and the agents ignore each other except when avoiding collisions.

Table I shows how the parameters of the mediator relate to previous work using a leader and a predator [6]. The first two rows in the table indicate the parameters used in prior models, and the last two rows indicate parameters for the two types of mediators introduced in this paper.

\begin{tabular}{|c||c|}
\hline Informed Agent & Order of Nominal Agent's Each Zone \\
\hline \hline Leader & $R^{\text {rep }} \leq R^{\text {ori }}<R^{\text {att }}<R^{\text {lead }}$ \\
\hline Predator & $R^{\text {rep }} \leq R^{\text {ori }}<R^{\text {att }}<R^{\text {pred }}$ \\
\hline Mediator & $R^{\text {rep }} \leq R^{\text {ori }}<R^{\text {pred }}<R^{\text {att }} / 2$ \\
\hline
\end{tabular}

TABLE I. How NOMINAL AGENTS ARE INFLUENCED. TOP TWO INFORMED AGENT INDICATE PREVIOUS MODELS.

\section{SHAPING SWARMS}

If we place more than one mediator in the center of a swarm, we can make the nominal agents track many different perimeter shapes. Before doing so, we note that a portion of this section and the previous section were first described in our previous work [15]; this paper significantly extends prior work and includes a careful user study with results that were not previously published. If multiple mediators are given a specific initial configuration and move with the same direction and the same speed, the shape of the swarm is approximately static as the group translates to a new location.

In order to create a range of controllable torus shapes, we alter nominal agent behavior to create so-called smart agents [15]. Smart agents, or S-agents, are inspired by the behavior of the sheep illustrated in Fig. 3. In this figure, the sheep are orbiting a moving car. Because the car covers part of the sheep's vision, the sheep cannot see the entire group's movement. Rather, they can see only the neighbors in front of them so they follow those neighbors.
Likewise, if S-agent $i$ in a swarm observes a set of neighbors $O$, it decides to follow the closest neighbor $E_{i}$ where

$$
E_{i}=\underset{j \in O}{\operatorname{argmin}}\left(\sqrt{\left(i_{x}-j_{x}\right)^{2}+\left(i_{y}-j_{y}\right)^{2}}\right) .
$$

This corresponds to a nearest neighbor topology which has been shown to accurately model interactions in natural flocks [16]. The main difference between an S-agent and a nominal agent is that an S-agent has a more narrow field of view $\left(\theta=180^{\circ}\right.$ rather than $\left.270^{\circ}\right)$. Because an $\mathrm{S}$-agent has a larger blind spot, it needs more than just attraction to maintain the connectivity of the swarm.

In order to make the agents "smarter", each agent $i$ remembers the last location of its closest neighbor, $P_{E_{i}}$. When an S-agent does not observe any neighbors within its vision, the S-agent recalls the last location of its closest neighbor and moves towards that location. As soon as the S-agent

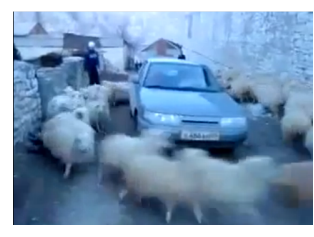

Fig. 3. Sheep's encircling motion around a car. observes a neighbor, it responds to the observed neighbor and resets its memory.

Another way of being smarter to maintain connectivity is that each S-agent has the ability to increase its speed when it gets far from its nearest neighbor [17]. The speed for agent $i$ is

$$
s_{i}(t+1)= \begin{cases}\gamma \times s & \text { if }\left\|P_{i}-P_{E_{i}}\right\|>\text { Stable Dist } \\ s & \text { otherwise }\end{cases}
$$

where $s$ is constant and $\gamma>1$ determines how much the agent increases its speed. We tested that when Stable Dist $\leq$ $0.9 \times R^{a t t}$ and $\gamma \geq 1.1$, the torus remained stable during the simulations. Also, we found that the mediator needs to move slower with S-agents than with nominal agents to maintain a stable torus formation.

Fig. 4 illustrates the different topologies that result when using mediators with either nominal agents or S-agents. Nominal agents show more influence dependencies than S-agents. This means that nominal agents respond to more neighbors than Sagents do in order to maintain their connectivity-S-agents need only the closest neighbor in front of them. As noted in [15], using S-agents slides the weight of control from being highly weighted on nominal agents to being equally weighted between mediators and S-agents. Because of this change, Sagents are better suited to shaping swarms than nominal agents.
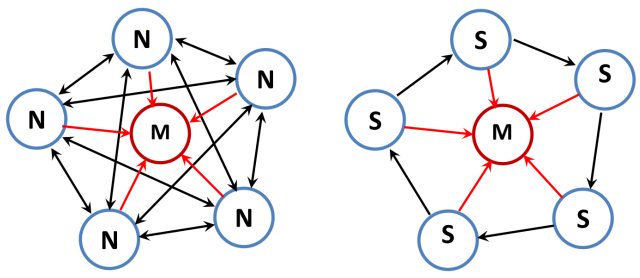

Fig. 4. Topologies among nominal agents $N$, S-agents $S$, and mediators $M$. Black lines indicate the response among nominal agents. Red lines indicate the response to the mediator. The notation $a \rightarrow b$ means $a$ is influenced by $b$. 
Fig. 5 illustrates that a group of S-agents under the influence of a group of mediators can adopt a set of very flexible shapes, more than is possible with nominal agents under the influence of a group of mediators. The next section presents results from an experiment that illustrate that $\mathrm{S}$-agents can be managed by mediators to perform interesting problems, and do so better than leaders or predators.

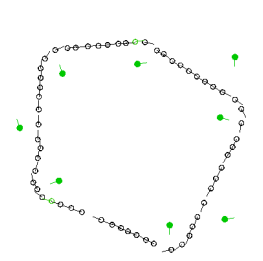

(a)

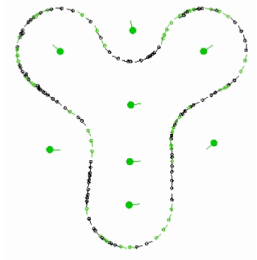

(b)
Fig. 5. Dynamic transformation of S-agents from an amorphous blob (a) to a Y shape (b) under the influence of a group of coordinating mediators.

\section{EXPERIMENT SETUP}

In this paper, we claim that $(a)$ mediator-control increases the manageability of swarm control compared to leaders and predators and (b) swarm-shaping can be used for a real application. To explore how swarm-shaping can be effectively used in a real world application, we designed an experiment that used a problem that is best performed when multiple robots can be placed in a flexible, dynamic shape around the perimeter of an interesting spatio-temporal problem. This means that task performance needs to be associated with the spatial allocation of robots under time pressure. We created an ocean-based oil spill scenario for the experiment since currents and winds cause the oil spill to take various shapes. This means that the robots need to be able to adopt different shapes to confine the oil contaminants.

We created two scenarios that subjectively have different workloads. This allows us to explore whether advantages of mediator-based control are robust to changes in the problem caused by environmental conditions. The experiment is thus a $2 \times 3$ design with two workload levels and three types of informed agent; see Table II.

\begin{tabular}{|c||c|c|c|}
\hline \multicolumn{1}{|c||}{} & \multicolumn{3}{c|}{ Informed Agent } \\
\hline Scenario & Leader & Predator & Mediator \\
\hline \hline Low Workload & & & \\
\hline High Workload & & & \\
\hline
\end{tabular}

TABLE II. THE LAYOUT OF THE CASE STUDY.

We include seven measurements that reflect two types of measures: manageability and task performance. Measures include both subjective and objective measures of performances. Measures of manageability are as follows:

- Sustainability: This is an objective measure of how well a human can sustain average influence over all agents in the collective. High sustainability indicates that it is easier for a human to manage the group. This can be calculated by $S_{t}=\sum_{i=1}^{t} B_{i}$ where, $S_{t}=$ sustainability at time $t, n=$ number of agents, and

$$
B_{i}= \begin{cases}1 & \text { if } d_{i} \leq R_{\text {influence }} \\ 0 & \text { otherwise }\end{cases}
$$

where, $d_{i}=$ distance between an agent $i$ and an informed agent (leader, predator, mediator), $R_{\text {influence }}=$ radius of influence zone, $B=$ the adjacency matrix between $\mathrm{S}$-agents and the informed agent.

- NASA-TLX: This is a subjective measure of the workload required to manage the group.

- Secondary task performance: This is a more direct measure of workload, because high error rates indicate that the human is using cognitive resources to manage the robots and has little free capacity.

- Travel distance: This indicates how far the informed agents (leader, predator, mediator) had to move, allowing us to infer how much effort the user requires to manage S-agents.

Measures of task performance are as follows:

- Amount of contaminant removed: The primary task is to surround a shaped contaminant by a group of agents, so this is a direct measure of performance.

- Contaminated area: The task is designed so that users can hypothetically remove the contaminants in three minutes, but this is difficult to do in practice. However, groups that are easy to control and shape should leave less contaminated area.

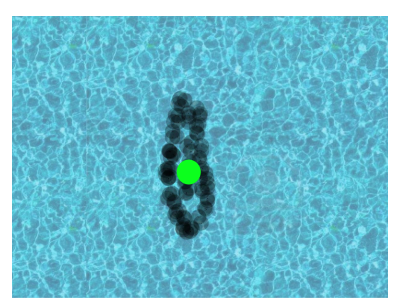

(a) Low workload scenario map.

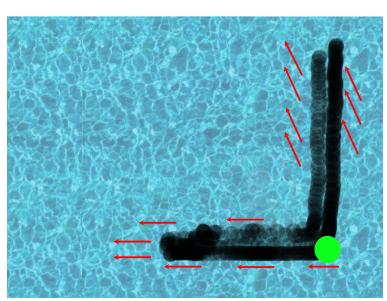

(b) High workload scenario map.
Fig. 6. The contaminant source (green dot) produces a new quanta of oil contaminant every two seconds. (a) No ocean currents. (b) Directions of ocean currents are marked as red arrows.

In the experiment, participants were given three minutes to remove as much contaminant as possible. Participants used a mouse and a keyboard to control leaders, predators, or mediators during the scenario. Parameter used in the experiment are shown in Table III. We measured distances in terms of units where $R^{r e p}$ is fixed as 1 unit because it is the minimum distance that is required to avoid collisions. All conditions fixed the influence range of informed agents (leaders, predators, mediators) at 14 units, the number of informed agents at 4 , and the number of nominal agents at 100 .

\begin{tabular}{|c||c|c|c|c|c|c|}
\hline \multicolumn{1}{|c||}{} & \multicolumn{6}{c|}{ Parameters } \\
\hline Agent Type & $R^{\text {rep }}$ & $R^{\text {ori }}$ & $R^{\text {att }}$ & s/unit & $\omega\left({ }^{\circ} /\right.$ sec $)$ & $\theta$ \\
\hline \hline Nominal Agent & 1 & 4 & 20 & 3 & 40 & 270 \\
\hline S-Agent & 1 & 1 & 20 & 4 & 40 & 180 \\
\hline
\end{tabular}

TABLE III. SUMMARY OF UNINFORMED AGENTS' PARAMETERS: SPEED $s$, TURNING RATE $\omega$, AND VISION RANGE $\theta$.

\section{MISSION}

Each participant operates each of the different types of informed agents (leader, predator, mediator) to manage and 
control multiple uninformed agents to form a perimeter around the oil spill and absorb as much oil as possible. As Fig. 6 shows, one oil source is located in the middle of the oil contaminants and produces a new quanta of oil contaminant every two seconds. Each quanta is repelled by other contaminants and moves depending on ocean currents. If the uninformed agents are near enough to the oil for long enough, the oil is absorbed ${ }^{1}$ Encircling a quanta makes the quanta disappear more quickly because encircling optimizes the number of agents within decontamination range.

For the secondary task, participants hear two different sounds: a target bell sound ("ding") and a distractor spring sound ("sproing"). They were instructed to press the space bar when they heard the bell and to do nothing when they heard the spring. Every two seconds, the probability $P$ of a sound playing is drawn from a uniform distribution, $u(0.3,0.7)$. The probability of a bell sound playing is fixed from the beginning of each scenario and is a Bernoulli random variable $R$, where $R \sim u(0.55,0.75)$.

Each participant was assigned to a counterbalanced combination of each scenario and informed agent yielding a withinsubjects designs. We recruited 13 participants from the campus of the Brigham Young University, 8 males and 5 females, ranging from 18-32 years old (average 24.23). After completing the informed consent process but before we gathered data, every participant was trained to manage the swarms with each type of informed agents in a simplified version of the oil spill problem.

\section{RESUlTS AND Discussion}

In this section, we address the following two questions: First, does mediator-control method improve the manageability of a robot swarm? Second, does mediator-control method increase the performance of decontamination task? Data was analyzed using a repeated measures ANOVA.

\section{A. Manageability}

Since the probability of playing sounds are random, it is hard to define how well participants did in the secondary task. Thus, we calculated the secondary task score by adding all the number of positive responses and negating the number of negative responses. Then, we normalized the score by the total number of produced sounds. Although averages show that the score for mediators is a little higher than others (see Table IV), the ANOVA for the secondary task revealed that there is no significant difference across the scenarios $(F[1,24]=0.983, p=0.412)$ and among the three informed agents $(F[2,36]=0.682, p=0.514)$.

For the score of NASA-TLX across the scenarios for predators $(F[1,24]=16.616, p=0.002)$ and leaders $(F[1,24]=7.014, p=0.02)$, there are significant differences. However, mediator showed no significant difference $(F[1,24]=0.226, p=0.643)$ across the scenarios. Fig. 7 illustrates that workload increases when using leaders and may actually decrease using predators, but stays relatively flat for

${ }^{1}$ Each quanta contains 3000 particles inside a circle of radius 5 units. Particles are absorbed at a rate one particle per simulation time step per agent within 5 units of the circle boundary. mediators. It also shows that the mediator's NASA-TLX score is the lowest.

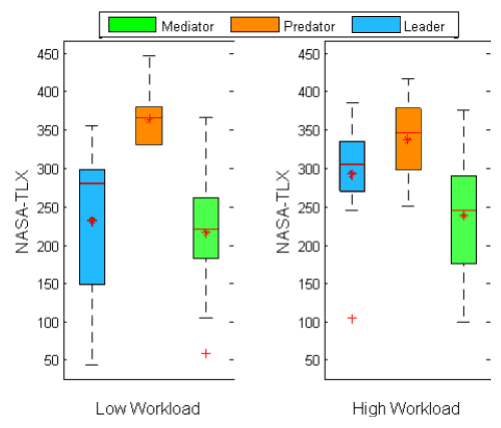

Fig. 7. NASA-TLX scores

Based on results from [18], we hypothesized that high sustainability enables a human to manage a swarm easily. However, Table IV shows that the leader has the highest sustainability. On the other hand, the mediator's sustainability is similar to the predator's because when the shape gets bigger, uninformed individual has more chance to interact each other and less chance to interact with mediators. This suggests that sustainability, meaning the total number of uninformed agents influenced by the informed agents, is less important for problems where a swarm must be shaped than for a swarm that must flock to different locations.

\begin{tabular}{|c||c|c|c|}
\hline & Leader & Predator & Mediator \\
\hline Secondary task score & 1.71 & 1.69 & 1.75 \\
\hline Sustainability & 176.2 & 17.92 & 17.71 \\
\hline Travel Dist.(unit) & 889.54 & 1054.97 & 249.0 \\
\hline
\end{tabular}

TABLE IV. QUALITATIVE RESULTS OF SECONDARY TASK SCORE, SUSTAINABILITY, AND TRAVEL DISTANCE.

However, the total distance traveled by the mediators' is much lower than the distances of other informed agents as shown in Table IV. As illustrated in Fig. 8, it is easy to see that mediators need to move less to manage the swarm than other two informed agents. This impacts the strategies used to control the uniformed agents. Because leaders facilitate sustainable influence, participants tended to gather all agents in the swarm near the contaminants and then guide this cluster from contaminant to contaminant. For predators, participants tended to turn the swarm into several groups of flocks and tori, which they then "pushed" to different contaminant areas; the predators were then moved around the map to guide the separate groups. Because of this, predators tend to travel a lot. Participants tended to place mediators near the boundaries of the spill and then move the mediators toward the source of the spill as contaminants were removed, resulting in little travel distance.

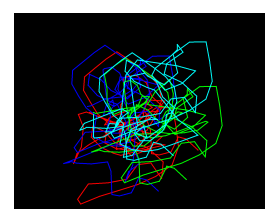

(a) Leaders

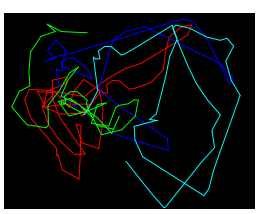

(b) Predators

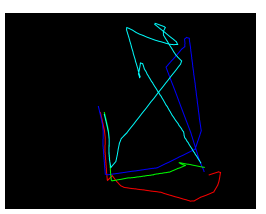

(c) Mediators
Fig. 8. Trajectories of informed agents.

In summary, lower NASA-TLX scores and lower distances travel suggest that mediator-based control is easier for humans. 


\section{B. Performance}

As shown in Fig. 9, both leader-based control $(F[1,24]=$ 4.457, $p=0.06)$ and predator-based control $(F[1,24]=$ 1.615, $p=0.23$ ) do not show significant differences in the amount of contaminant removed, but mediator-based control $(F[1,24]=9.523, p=0.009)$ shows a significant difference across the scenarios, suggesting that mediator-based control scales better with workload. Importantly, mediators outperform leaders and predators, which is not surprising since we designed the scenarios to require swarm control compatible with mediator-based influence. Moreover, because the initial condition of high workload scenario includes more oil contaminant than the initial amount of oil contaminant in low workload scenario, removed contaminant score for the mediator method was increased under high workload conditions.

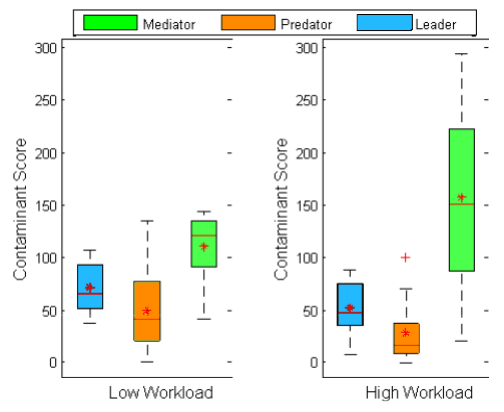

Fig. 9. Removed contaminant scores for each scenario.

The amount of area that is contaminated after three minutes shows no significant difference across the scenarios, but there are significant differences among the informed agents $(F[2,36]=46.235, p<0.001)$. Fig. 10 illustrates that mediators tend to gather the oil contaminant into one place and prevent the oil from expanding over the ocean surface.

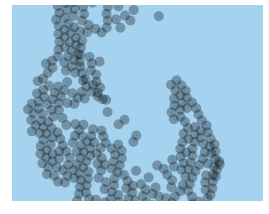

(a) Leader

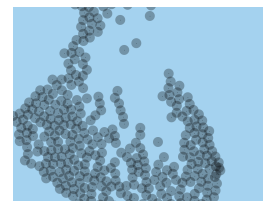

(b) Predator

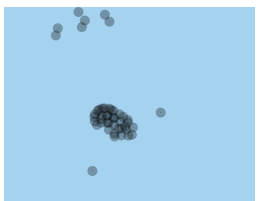

(c) Mediator
Fig. 10. Contaminated area in the map of each informed agent.

These two measures indicate that the mediator-based control performs better than either leader- or predator-based controls for this task.

\section{CONCLUSION AND FUTURE WORK}

We introduced a mediator-based control model for humanswarm interaction and demonstrated that this model can be used to transform a swarm into a variety of shapes. Because mediator-based swarm control allows a swarm to maintain a torus formation while it is moving, the swarm retains the advantages of torus behavior, in contrast to previous work on leader- and predator-based control. We also investigated how the mediator-based control is better in managing swarms and performing decontamination task. Future work will study whether mediated swarms can be robustly applied to real robots. Future work will also examine how robustly the mediator model can handle a variety of shapes.

\section{ACKNOWLEDGMENT}

We appreciate the Science of Autonomy program of the Office of Naval Research for funding this work. The opinions of this paper do not necessarily reflect the funding agency.

\section{REFERENCES}

[1] R. Olfati-Saber, "Flocking for multi-agent dynamic systems: Algorithms and theory," IEEE Trans on Automatic Control, vol. 51, no. 3, pp. 401420, 2006.

[2] I. Couzin, J. Krause, R. James, G. Ruxton, and N. Franks, "Collective memory and spatial sorting in animal groups," J. of Theoretical Biology, vol. 218, no. 1, pp. 1-11, 2002.

[3] C. Reynolds, "Flocks, herds and schools: A distributed behavioral model," in ACM SIGGRAPH Computer Graphics, vol. 21, no. 4. ACM, 1987, pp. 25-34.

[4] R. Wiegand, M. Potter, D. Sofge, and W. Spears, "A generalized graphbased method for engineering swarm solutions to multiagent problems," Parallel Problem Solving from Nature-PPSN IX, pp. 741-750, 2006.

[5] L. Alboul, J. Saez-Pons, and J. Penders, "Mixed human-robot team navigation in the guardians project," in IEEE Intl. Wkshop. on Safety, Security and Rescue Robotics, 2008. IEEE, 2008, pp. 95-101.

[6] A. Goodrich, P. Sujit, S. Kerman, B. Pendleton, and J. Pinto, "Enabling human interaction with bio-inspired robot teams: Topologies, leaders, predators, and stakeholders." Technical Report BYU-HCMI Technical Report 2011-1, Brigham Young University, Tech. Rep., 2011.

[7] A. Wood and G. Ackland, "Evolving the selfish herd: emergence of distinct aggregating strategies in an individual-based model," Proc. of the Royal Society B: Biological Sciences, vol. 274, no. 1618, pp. 16371642, 2007.

[8] Z. Kira and M. Potter, "Exerting human control over decentralized robot swarms," in 4th Intl. Conf. on Autonomous Robots and Agents, 2009. IEEE, 2009, pp. 566-571.

[9] H. Su, X. Wang, H. Dong, and G. Chen, "Flocking with multiple virtual leaders based on position measurements," in 7th Asian Control Conf., 2009. IEEE, 2009, pp. 1156-1161.

[10] M. Mabrouk and C. McInnes, "Swarm robot social potential fields with internal agent dynamics," in 12th International Conference on Aerospace Sciences and Aviation Technology, ASAT-12, 2007.

[11] G. Elkaim and R. Kelbley, "A lightweight formation control methodology for a swarm of non-holonomic vehicles," in Aerospace Conf. IEEE, 2006, pp. 8-pp.

[12] B. Varghese and G. McKee, "Investigating feasible tools for swarm pattern transformation," in Second Intl. Conf. on Robot Communication and Coordination, 2009. IEEE, 2009, pp. 1-7.

[13] H. Kawashima, G. Zhu, J. Hu, and M. Egerstedt, "Responsiveness and manipulability of formations of multi-robot networks," in 2012 IEEE 51st Annual Conference on Decision and Control. IEEE, 2012, pp. 4622-4628.

[14] A. Kolling, S. Nunnally, and M. Lewis, "Towards human control of robot swarms," in Proceedings of the seventh annual ACM/IEEE intl. conf. on Human-Robot Interaction. ACM, 2012, pp. 89-96.

[15] S.-Y. Jung, D. S. Brown, and M. A. Goodrich, "Shaping couzinlike torus swarms through coordinated mediation," in Proceedings of IEEE International Conference on Systems, Man, and Cybernetics, Manchester, To appear., 2013.

[16] M. Ballerini, N. Cabibbo, R. Candelier, A. Cavagna, E. Cisbani, I. Giardina, V. Lecomte, A. Orlandi, G. Parisi, A. Procaccini et al., "Interaction ruling animal collective behavior depends on topological rather than metric distance: Evidence from a field study," Proc. of the National Academy of Sciences, vol. 105, no. 4, p. 1232, 2008.

[17] N. Bode, D. Franks, and A. Wood, "Limited interactions in flocks: relating model simulations to empirical data," J. of The Royal Society Interface, vol. 8, no. 55, pp. 301-304, 2011.

[18] M. A. Goodrich, B. Pendleton, S. Kerman, and P. B. Sujit, "What types of interactions to bio-inspired robot swarms and flocks afford a human?" in Proceedings of Robotics Science and Systems, Sydney, Australia, June 2012. 